\title{
L'AFRIQUE DU COTON À CANCÚN : LES ACTEURS D'UNE NÉGOCIATION
}

\begin{abstract}
FIN AVRIL 2003, QUATRE PAYS AFRICAINS DÉPOSAIENT UNE PROPOSITION DE NÉGOCIATION À L'OMC INTITULÉE «RÉDUCTION DE LA PAUVRETÉ: INITIATIVE SECTORIELLE SUR LE COTON". QUELQUES MOIS APRÈS, LE «DOSSIER COTON " DÉFRAYAIT LA CHRONIQUE PENDANT LA CONFÉRENCE De Cancún, ILlustrant de manière emblématique les CONTRADICTIONS ENTRE PAYS INDUSTRIALISÉS ET PAYS EN DÉVELOPPEMENT

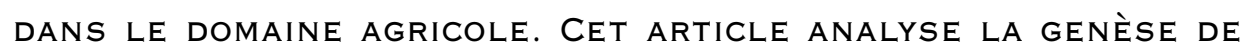
CET ÉVÉNEMENT ET MET EN ÉVIDENCE LES JEUX D'ACTEURS QUI Y ONT CONDUIT.
\end{abstract}

Fin avril 2003, le Bénin, le Burkina Faso, le Mali et le Tchad déposaient une proposition de négociation à l'Organisation mondiale du commerce $(\mathrm{OMC})$ intitulée « Réduction de la pauvreté: initiative sectorielle sur le coton». En septembre 2003, lors de la conférence ministérielle de l'OMC à Cancún, l'Afrique défrayait la chronique sur le «dossier coton». Portedrapeaux de l'Afrique de l'Ouest et du Centre (AOC), les quatre pays soumissionnaires illustrent de manière emblématique les contradictions liées aux conditions actuelles de la production de coton dans le monde ${ }^{1}$ et, audelà, les conditions de l'insertion internationale des pays africains dans un monde libéralisé.

L'asymétrie de la configuration de l'économie cotonnière est flagrante. D'un côté, les quatre pays de $\mathrm{l}^{\prime}$ «Initiative coton » produisent près de un million de tonnes et représentent $17 \%$ du marché mondial en 2002 (contre $4 \%$ en 1980); le coton contribue entre 30 et $40 \%$ de leurs recettes d'exportations et il fait vivre environ 10 millions de personnes. Il est reconnu comme l'une des productions compétitives de l'Afrique ${ }^{2}$ et peut être considéré comme l'une des rares success stories du continent. D'un autre côté, les deux grandes puissances économiques que sont les États-Unis (EU) et l'Union européenne (UE) perturbent le marché mondial en subventionnant leurs producteurs de coton. Les EU représentent $40 \%$ du commerce mondial de coton et distribuent $68 \%$ des subventions pour seulement 25000 producteurs $^{3}$. L'UE, même si elle n'exporte pas de coton, accorde $18 \%$ des subventions mondiales pour seulement 2,5\% de la production. D'autres pays subventionnent aussi leur production cotonnière (comme la Chine et la Turquie) mais selon des modalités moins bien connues et qui, a priori, ne perturbent pas le marché mondial 4 .

La déprime des cours du coton en 2001 est, selon les analystes, largement imputable à la politique de subvention américaine aux 
producteurs de coton ${ }^{5}$. La disproportion des chiffres et l'iniquité flagrante mise en évidence par leur comparaison ont permis au dossier africain de bénéficier aisément d'une très large sympathie et de nombreux soutiens lors de la conférence de Cancún. Même si le facteur de blocage principal a été le refus des pays en développement de débattre des «questions de Singapour ${ }^{6}{ }$, les désaccords sur le dossier agricole en général et sur le coton en particulier auront mobilisé les énergies pendant la conférence. Pour la première fois, une négociation commerciale internationale achoppait sur une revendication africaine reconnue comme légitime par la plupart des autres pays.

Comment en est-on arrivé à cette situation sans précédent? Avec ce dossier coton, l'Afrique a-t-elle marqué une nouvelle étape dans la construction de ses capacités de négociation internationale? Quels sont les jeux d'acteurs qui ont rythmé le processus de préparation de la conférence de Cancún? Quel est le point de départ de ce processus? Les Africains ne se sont-ils pas laissé entraîner dans une croisade libérale contre les subventions agricoles? Et aujourd'hui, un an après Cancún, quelles perspectives concrètes pour les pays africains et pour leurs producteurs de coton? Quelles leçons tirer en termes d'analyse des jeux d'acteurs dans les négociations internationales?

Dans un premier temps, nous examinerons succinctement la pertinence relative de la thèse de l'instrumentalisation pour comprendre ce qui s'est passé a priori à Cancún. Ensuite, un examen de la genèse de la conférence et de la construction du dossier coton permettra $\mathrm{d}$ 'analyser plus finement les jeux d'acteurs et montrera les enchaînements d'événements qui ont conduit le dossier coton, initialement ouvert par des représentants de producteurs, sur la scène des négociations commerciales internationales avec l'entrée en lice d'organisations non gouvernementales (ONG) altermondialistes, de firmes cotonnières et de pays agro-exportateurs libéraux. Enfin, quelques enseignements seront tirés sur la manière d'analyser les processus de construction d'alliances et de jeux «multi-acteurs ».

\section{LE COTON, UN DOSSIER EN TROMPE-L'CEIL?}

Pour analyser ce qui s'est passé, il faut considérer que derrière l'apparente force de la position africaine se dissimulent des logiques d'instrumentalisation croisées qui visaient, derrière l'Afrique et le coton, d'autres buts.

À certains égards, le dossier coton peut apparaître comme le "cheval de Troie» de combats plus larges ou d'une autre nature. D'un côté, plusieurs pays connus pour leurs positions libérales ${ }^{7}$ ont rapidement soutenu les positions africaines, en particulier celle concernant la suppression des subventions agricoles. C'est d'ailleurs cette menace qui semble expliquer certaines des réticences de l'UE à s'engager fermement dans un soutien du dossier coton à Cancún, craignant des critiques trop virulentes sur le processus de réforme de la Politique agricole commune (PAC). D'un autre côté, des organisations de la société civile vont s'emparer du dossier coton, en y voyant un cas d'école idéal pour souligner les incohérences entre politiques commerciales et politiques d'aide au développement de l'UE et des EU. Le coton est aussi, pour plusieurs ONG, un support de communication parfait pour questionner la légitimité des instances internationales en charge de la régulation du commerce et du développement économique. 


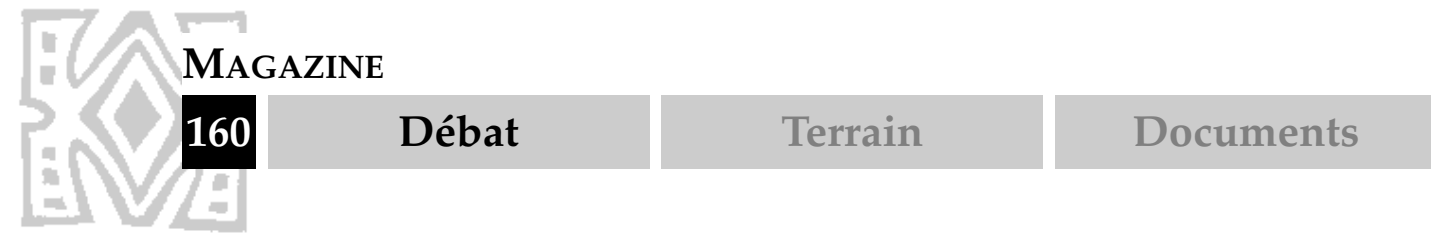

Pour les États africains, le dossier coton aura été une bonne occasion de redorer leur blason. Les gouvernements d'AOC et leurs représentants sont apparus sur la scène internationale comme les hérauts de la lutte contre la pauvreté et du soutien à leurs populations, alors que les exemples ne manquent pas quant à leurs défaillances dans le développement économique et social de leurs pays.

L'analyse de la genèse du dossier coton illustre l'informalisation croissante de ces sociétés qui voit des organisations de producteurs, des ONG, des sociétés cotonnières se coaliser pour faire entendre des revendications, certes légitimes, mais qui sont de nature à affaiblir de l'intérieur la crédibilité de l'État en Afrique. La stigmatisation d'un ennemi extérieur et la détermination d'un objectif simple (les subventions américaines) permettent aux États du continent de faire l'économie d'une analyse de leurs propres responsabilités dans les difficultés que peuvent rencontrer les filières cotonnières africaines. Finalement, en s'emparant, tardivement, d'un combat initialement porté par les organisations de producteurs de coton, les États de la région montrent leur capacité à reconfigurer leur action dans le cadre général de leur privatisation ${ }^{8}$. Mais, surtout, ils témoignent de leurs difficultés à être «proactifs » et à proposer leurs propres ordre du jour et vision du développement.

Dans d'autres cas, les dirigeants africains bâtissent des discours anticipateurs correspondant, de fait, au refus d'une analyse du présent, comme c'est le cas avec le Nouveau Partenariat économique pour le développement de l'Afrique (Nepad) dont les promoteurs appellent de leurs vœux de nombreuses initiatives privées pour développer des biens publics en Afrique, marquant, de facto, l'incapacité des États à assurer leurs fonctions régaliennes ${ }^{9}$.

Même s'il paraît difficile d'établir la thèse de l'instrumentalisation de l'Afrique à Cancún, on ne peut que reconnaitre que les États africains, fragilisés par vingt-cinq années d'ajustement structurel ${ }^{10}$, se trouvent en position de faiblesse vis-à-vis des grandes puissances dans les négociations du commerce international, mais aussi envers les États émergents (regroupés au sein du G-2111 à Cancún) dont la base productive et la capacité de réaction vis-à-vis des signaux du marché mondial sont sans commune mesure avec les maigres atouts des économies africaines, en tout cas à court terme. Ainsi, pris dans l'étau de puissances commerciales à la recherche de l'accroissement de leurs parts de marché mondial et de gouvernants en quête de légitimité, les producteurs africains de coton paraissent, à première vue, avoir joué à Cancún une partition dont ils ne maîtrisaient pas forcément les notes. Cependant, dans les faits, il convient de relativiser la thèse de l'instrumentalisation des producteurs africains de coton et de se méfier des apparences. En effet, un examen approfondi du processus menant à Cancún montre la complexité des jeux entre acteurs et permet d'appréhender, sous un jour nouveau, l'évolution des rapports entre, d'une part, États et sociétés civiles en Afrique ${ }^{12}$, et, d'autre part, les pays du Sud et ceux du Nord. Ainsi, accorder une importance déterminante aux jeux d'instrumentalisation dans l'analyse peut conduire à occulter à la fois le renforcement des acteurs africains engagés dans le processus qui a contribué à l'échec de la conférence de Cancún et les logiques d'apprentissage permises par la confrontation entre acteurs différents. 
LA LENTE GENÈSE DU DOSSIER COTON

Les producteurs africains de coton ont été les premiers, en novembre 2001, à investir l'espace international par une déclaration dénonçant l'effet négatif des subventions américaines et européennes sur les cours du coton $^{13}$. Sous l'impulsion de l'Union nationale des producteurs de coton du Burkina Faso (UNPCB), d'autres organisations de producteurs (Bénin, Mali puis Cameroun et Madagascar début 2002) s'engagent sur la scène internationale et interpellent leurs gouvernements respectifs. Cette déclaration insiste sur les contradictions entre politiques commerciales et politiques de développement: «Au moment où il est question de lutter contre la pauvreté, les producteurs de coton de l'Afrique de l'Ouest ont tout de suite compris que ce n'est qu'au prix de leurs efforts qu'ils peuvent venir à bout de cette pauvreté. Ils se sont mis à la tâche, et au moment où ils obtiennent un nouveau record de production, voilà que subitement les cours du coton s'effondrent. Nous en arrivons à nous interroger sur la volonté réelle des pays riches à faire reculer la pauvreté dans les pays pauvres ${ }^{14}$.» On remarquera que cette déclaration commune, diffusée grâce à Internet, est la première expérience réussie de «cyberpétition» pour des organisations de producteurs en Afrique.

À peu près au même moment, en février 2002, un travail réalisé en 2001 par deux $\mathrm{ONG}^{15}$ sur la durabilité des filières coton en Afrique permet de réunir des représentants de producteurs de coton à Dakar. Lors de cette rencontre, des contacts sont noués entre organisations de producteurs (OP) et ONG: leur objectif est d'être plus présents dans les réunions internationales sur le coton pour faire connaître les positions des producteurs.
Les $\mathrm{OP}$ et les ONG se font inviter à une réunion organisée à Lomé par la Banque ouest-africaine de développement (BOAD) sur l'avenir de la filière coton, mais les représentants de producteurs ne sont alors qu'au nombre de 3 pour 180 participants.

Une première remarque s'impose sur les mécanismes de représentation des producteurs africains, à travers la médiation de leurs organisations. Schématiquement, on peut identifier au sein du mouvement paysan ouest-africain des tensions entre, d'une part, une logique verticale, mettant en avant la défense d'intérêts spécifiques, souvent structurés autour d'un produit et selon une logique économique (ou logique de filières), et, d'autre part, une logique horizontale, transversale, visant à défendre et promouvoir un modèle d'agriculture basé sur l'exploitation familiale (vision défendue par le Roppa ${ }^{16}$ ). Ces deux logiques sont inhérentes à la création de la plupart des organisations de producteurs et le mot «tensions» renvoie ici à une dynamique de construction et non d'opposition binaire entre des logiques qui seraient incarnées par des organisations ou des individus bien identifiés ${ }^{17}$.

La Conférence des ministres de l'Agriculture d'Afrique de l'Ouest et du centre (CMA/AOC) organise une réunion en juin 2002 à Abidjan : le dossier coton commence à prendre forme, avec une implication des représentants de producteurs épaulés par les ONG. À l'époque, les protestations des producteurs africains ne sont pas prises au sérieux au niveau international, et les gouvernements africains sont divisés sur l'attitude à avoir vis-à-vis des pays riches: attaquer les États-Unis à l'OMC en portant plainte à l'ORD (Organe de règlement des différends), au risque de représailles via les accords de coopération (notamment l'AGOA) ${ }^{18}$, 
ou chercher à négocier directement. C'est finalement cette seconde option qui est retenue avec, fin juin 2002, à Abidjan, un mandat confié à la CMA / AOC pour analyser l'impact des subventions sur les filières cotonnières et négocier avec les EU et l'UE. À la suite de la réunion d'Abidjan, l'Association cotonnière africaine (ACA) voit le jour et regroupe les principales sociétés cotonnières de la sousrégion. La formulation d'un objectif clair sur le plan international (supprimer les subventions liées au coton) facilite des alliances inédites entre les sociétés cotonnières et certains représentants des producteurs ouest-africains membres du Roppa, pourtant très méfiants envers les logiques de filières ${ }^{19}$.

Le processus s'accélère en septembre 2002 avec la plainte déposée par le Brésil contre les EU sur les subventions cotonnières et la sortie d'un rapport accablant préparé par Oxfam International ${ }^{20}$. En plus de la CMA/AOC, l'Uemoa (Union économique et monétaire ouest-africaine) et la Cedeao (Communauté économique des États de l'Afrique de l'Ouest) se mobilisent pour réaliser des études similaires qui détaillent l'impact des subventions sur la production cotonnière africaine. Le Burkinabè Ablasse Ouedraogo, qui a terminé son mandat de directeur général adjoint de l'OMC en novembre 2002, propose ses services à l'Uemoa sur ce dossier. Un bureau genevois, IDEAS 21, présidé par Arthur Dunkel (ancien directeur général du GATT [Accord général sur les tarifs douaniers et le commerce]), assure l'expertise auprès des négociateurs africains à Genève: celle-ci conduit à la soumission par les quatre pays africains de l' «Initiative coton » à l'OMC, qui est engagée le 30 avril 2003 avec le soutien de la Cedeao dans un premier temps, puis, plus tardivement, de l'Uemoa.
Cet engagement tardif des gouvernements africains peut être compris comme le résultat d'une pression croissante et médiatisée des producteurs de coton, appuyés par les ONG, qui ont su utiliser les scènes sous-régionales africaines pour amplifier leurs messages. Représentants de producteurs et $\mathrm{ONG}^{22}$ parviennent à tisser un réseau de soutiens associant une expertise diversifiée et reliée à des bureaux genevois spécialisés dans l'accompagnement des pays en développement dans les négociations commerciales (le « groupe de Genève »): le lien est fait avec les négociateurs africains auprès du siège de l'OMC. En dépit d'une certaine volonté des représentants africains auprès de l'OMC, on soulignera la fragilité du dispositif puisque seuls deux des quatre pays disposent d'un ambassadeur permanent à Genève: le Bénin, qui assurera la coordination des quatre pays, et le Mali, le Burkina Faso mobilisant son ambassadeur permanent à Bruxelles sur ce dossier. Par ailleurs, la nécessité pour ces ambassadeurs d'en référer à leur capitale pour certaines décisions politiques complexifiera d'autant le travail tout en limitant les capacités de réaction.

Pourtant, l'unité africaine derrière le dossier coton cache mal des rivalités qui vont croître à l'approche de la conférence. En juin 2003, la restitution de l'étude Uemoa est l'occasion de tensions fortes entre experts sur l'attitude à adopter deux mois avant Cancún. L'enjeu est le contrôle de l'expertise auprès des négociateurs africains sur le dossier coton. Ces rivalités se nourrissent aussi de clivages sensibles, notamment autour d'un éventail de positions allant d'options libérales ou, au contraire, altermondialistes, en passant par des attitudes plus pragmatiques. Une dernière réunion de préparation est organisée à Saly 
(Sénégal) entre les différents acteurs africains en vue de bâtir une stratégie cohérente de défense du dossier coton à Cancún. Une autre tension est perceptible entre la Cedeao et l'Uemoa: la multiplicité des arènes sousrégionales et leur rivalité ont sans doute ouvert de nouveaux espaces pour les producteurs et les ONG, qui ont su utiliser les organisations régionales pour accroître la pression sur leurs États et consolider leurs positions.

Malgré ces fissures liées à la montée des tensions quelques mois avant le rendez-vous de l'OMC, l'Afrique et le coton sont au centre de la conférence de Cancún. Juste avant la conférence, un «cotton tour » est organisé en France, en Belgique, aux Pays-Bas puis aux États-Unis, avec un panel d'acteurs d'AOC. Ces rencontres permettent de diffuser les principaux éléments du dossier coton au grand public et d'amorcer un dialogue avec des représentants politiques. Une pétition de 250000 producteurs africains est remise aux ministres africains du Commerce qui se rendent à Cancún. La pression est ainsi maintenue sur les représentants des États qui devront défendre le dossier coton.

\section{Le blocage de Cancún}

Deux jours avant l'ouverture de la conférence, un "cotton day» est organisé sous la houlette du ministre allemand de la Coopération, qui permet aux délégations de tester leurs arguments et de commencer à interpeller les médias sur place ${ }^{23}$. Dès l'ouverture de la conférence, les quatre ministres des pays africains présentent la soumission coton faite à l'OMC. La délégation européenne se dégage de toute responsabilité en arguant qu'elle n'exporte pas de coton et ne dispose pas de mécanisme de soutien aux exportations. Les EU, principale cible des revendications du dossier coton, rejettent toute idée de concession sur les subventions et proposent une approche plus large englobant le coton et le textile. L'importance accordée à ce dossier est indéniable: le directeur général de l'OMC est invité par le président de la conférence-le ministre mexicain des Affaires étrangères, Luis Ernesto Derbez à conduire personnellement les consultations sur ce dossier avec l'ensemble des pays concernés. Des conférences de presse et plusieurs rencontres bilatérales ponctuent les quelques jours de la conférence; mais le projet révisé de déclaration ministérielle reprend pratiquement les propositions faites par la délégation américaine que les partenaires africains avaient pourtant rejetées en bloc ${ }^{24}$.

La première difficulté à laquelle s'est heurté le président de la conférence a concerné le choix du texte idoine pour les discussions: un contentieux, portant notamment sur le volet agricole, a opposé de nombreux pays en développement à l'UE et aux EU sur le projet de texte publié le 24 août par le secrétariat de l'OMC et devant servir de base aux négociations de Cancún. Pour sortir de l'impasse, un groupe de pays regroupés au sein du G-21, dont le Brésil, la Chine et l'Inde, soumet un nouveau texte, suivi d'un autre conçu par les pays africains. Une solution, qui se révélera par la suite périlleuse, est proposée par le président de la conférence, à savoir la division des négociations en cinq groupes distincts: agriculture, développement, accès aux marchés des produits non agricoles, «questions de Singapour», et autres questions. Les facilitateurs désignés pour présider chaque groupe de travail sont responsables de l'élaboration de projets provisoires relatifs à leurs groupes respectifs. Le résultat 
de cette organisation est la paralysie des négociations.

Le véritable théâtre des opérations s'est alors rapidement déplacé des salles de négociation vers des lieux de contacts informels, ce qui eut pour résultats concrets, d'une part, la constitution d'une nouvelle alliance (le G-90), réunissant les pays les moins avancés (PMA), les pays de l'Union africaine (UA) et les pays Afrique-Caraibes-Pacifique (ACP) et, d'autre part, la formation d'une coalition objective UE/EU faisant de l'ouverture des discussions sur les questions dites «de Singapour», le préalable à toute éventuelle concession sur d'autres sujets, notamment l'agriculture ${ }^{25}$.

$\mathrm{Au}$ regard de la rigidité des positions des différents groupes d'acteurs et du temps limité de la conférence (du 10 au 14 septembre 2003), cette dernière ne pouvait que déboucher sur une impasse, dont l'appréciation fut contingente aux objectifs annoncés, aux espérances cachées et éventuellement aux arrière-pensées des différents acteurs.

\section{QUELS ENJEUX APRÈs CANCÚN?}

Après Cancún, le coton est retenu comme une des quatre questions prioritaires à traiter par l'OMC. Deux divergences principales ressortent de la situation actuelle. En premier lieu, il y a désaccord sur le fait d'intégrer les questions cotonnières dans le dossier plus large des questions agricoles. Les EU et l'UE ${ }^{26}$ sont favorables à cette option alors que les pays africains s'y opposent tant, pour eux, cela risque de faire perdre au coton sa spécificité et de retarder des décisions concrètes. En second lieu, les discussions internationales opèrent une distinction entre questions commerciales et questions de développement. Ainsi, l'unité de l'Initiative africaine en faveur du coton se trouve écartelée entre le volet commercial (les subventions) et le volet développement (des engagements financiers en faveur des filières cotonnières). Là encore, les pays africains s'opposent à cette séparation alors que les organisations internationales, même si elles soulignent les liens entre les deux, en font un traitement différencié.

Paradoxalement, l'OMC, après avoir rejeté sur les institutions de Bretton Woods les questions de développement, prend l'initiative, en mars 2004, de tenir une réunion à Cotonou sur la filière coton. Cette volte-face illustret-elle le malaise de cette institution qui cherche à masquer l'immobilisme sur les questions commerciales?

Depuis Cancún, le coton continue de mobiliser certains acteurs mais plutôt sur le registre «développement»: la France puis l’Union européenne cherche à mobiliser la communauté des bailleurs de fonds pour réfléchir à des actions visant à renforcer les filières cotonnières africaines. Le Forum UE-Afrique sur le coton, qui s'est tenu à Paris début juillet 2004, a traité presque exclusivement de questions liées au développement des filières cotonnières, malgré l'insistante demande des États africains de trouver des pistes de solution aux questions commerciales. Alors que les représentants africains attendaient des signaux clairs d'un engagement européen sur le volet commercial, les autorités européennes et les bailleurs de fonds les ont surtout invités à utiliser les moyens financiers auxquels ils ont déjà accès dans le cadre de l'aide publique au développement, pour soutenir les filières cotonnières. Ainsi, ce forum aura surtout été l'occasion de débats sur des questions importantes pour l'avenir de la compétitivité des filières, notamment sur les mécanismes 
possibles de régulation du prix du coton et les biotechnologies 27 .

Fin juillet 2004, à Genève, un accord d'intention a été passé entre les EU et les pays africains attachés au dossier coton. Cet accord signe la fin de «l'exception coton » en réintégrant la question cotonnière dans le dossier plus général des négociations agricoles internationales. Contrairement à ce que proposait le Bénin, il ne précise pas d'objectifs chiffrés ni d'échéances, mais stipule simplement que le coton sera traité de manière «ambitieuse, rapide et spécifique » et qu'un «sous-comité du coton» se réunira régulièrement pour débattre de la question. L'événement africain de Cancún semble loin et tout se passe comme si chacun était retourné à ses occupations, guidé par ses «agendas» spécifiques: élections américaines, négociations agricoles internationales, renouvellement de la composition de la Commission européenne, etc.

Le dossier coton n'était-il qu'un feu de paille? Probablement oui sur le plan des résultats concrets à attendre suite à la démarche des pays africains avec leur Initiative en faveur du coton. Par contre, on peut émettre l'hypothèse que l'irruption du dossier coton dans le débat public international aura fait progresser l'idée que le secteur agricole nécessite des régulations que les seuls mécanismes d'un marché international libéralisé ne peuvent assurer. Les producteurs africains l'ont bien compris: lors d'une réunion du Roppa en mai dernier à Cotonou, ils soulignaient l'importance pour eux de s'assurer un développement de la filière coton dans le cadre plus large d'une amélioration et de la sécurisation du revenu des producteurs, en réduisant la dépendance de la filière du marché international au profit d'un marché régional. Les producteurs africains insistent aussi sur l'importance d'avoir une approche centrée sur l'exploitation familiale agricole dont il faut s'assurer le maintien du potentiel de production dans un souci du respect de l'environnement ${ }^{28}$. Ces prises de position témoignent d'une étape dans la construction du mouvement paysan africain avec un lien plus étroit, mais sans doute encore perfectible, entre la représentation des intérêts spécifiques autour d'un produit et la défense plus globale d'une vision d'avenir pour l'agriculture familiale en Afrique de l'Ouest. Au-delà du coton, le véritable enjeu est sans doute la capacité qu'auront les États africains (et plus largement les pays en développement) à se doter de politiques agricoles leur permettant de garantir un revenu décent à leurs agriculteurs tout en contribuant au développement économique ${ }^{29}$.

\section{QUELS ENSEIGNEMENTS TIRER DE CETTE EXPÉRIENCE?}

En conclusion, cette expérience illustre bien la limite d'interprétation ne prenant en compte que des acteurs à l'échelle nationale ou internationale: les États africains, l'Union européenne,... Plusieurs exemples démontrent les limites de ce type d'analyse de situations qui relèvent souvent de véritables jeux d'acteurs. Quelques exemples permettent d'illustrer cette idée. Plusieurs pays européens (France, PaysBas et Allemagne) ont joué un rôle actif pour tenter de trouver une issue positive au dossier coton à Cancún. Comme le précise un observateur, «l'Union européenne disposait d'une forte volonté en interne de faire des propositions concrètes mais cette démarche constructive de l'UE restera attendue jusqu'à la fin de la conférence ${ }^{30}$ ». La position de la France sur le dossier coton est souvent présentée comme favorable 
aux revendications africaines: cette attitude bienveillante ne doit pourtant pas masquer les réticences du ministère français de l'Agriculture, peu enclin à soutenir des positions suggérant intrinsèquement une suppression des subventions agricoles. L'engagement des États africains dans le dossier coton a été, pendant pratiquement toute l'année 2002, un des objectifs du travail de pression des producteurs de coton et des ONG. Ces mêmes États, en première ligne du combat international en faveur des filières cotonnières africaines, n'en demeurent pas moins ambigus dans leur comportement au sein des filières nationales ${ }^{31}$. Dans ces exemples, on voit bien que les positions de ces «macroacteurs» existent bel et bien, mais elles peuvent être considérées comme le fruit d'équilibres internes: en tant que positions visibles et officielles (à travers les déclarations ou communiqués), il est intéressant de les considérer aussi comme des enjeux en soi pour décrypter le travail d'influence des groupes d'intérêts ${ }^{32}$.

L'analyse de ce dossier coton illustre aussi l'importance de la prise en compte des différentes échelles géographiques à la fois comme espace de position des acteurs, mais aussi comme arène d'investissement dans les stratégies de défense des intérêts. Par exemple, l'espace sous-régional africain, avec sa multiplicité d'acteurs (Uemoa, Cedeao, CMA/ AOC,...), a été largement investi et utilisé par les producteurs et les ONG pour amener progressivement les États africains à s'engager sur le dossier coton. La France a cherché à convaincre l'Europe de l'importance d'adopter une position bienveillante et de porter le débat sur les questions de développement face à l'impossibilité de progresser sur les questions commerciales. On pourrait dire que certains acteurs peuvent être considérés comme des acteurs clés dans ce dossier (les organisations de producteurs, les $\mathrm{ONG}, . .$.$) , alors que d'autres acteurs sont$ plutôt des «acteurs-relais » ou des «acteurs cibles » qu'il s'agit soit de convaincre, soit d'amener à adopter une position attendue.

Enfin, l'analyse de la négociation suppose la prise en compte de la genèse des alliances et des jeux d'acteurs dans une optique synchronique. Beaucoup d'analyses privilégient des jeux de position entre acteurs sans prendre en compte la dimension historique de la construction des alliances, les logiques d'apprentissage entre acteurs lors des consultations et des négociations. Cependant, détachées des contingences matérielles et temporelles, les analyses diachroniques risquent aussi de passer à côté des ressorts de l'action collective et d'ignorer les mécanismes de renforcement des capacités intrinsèquement liés au processus de construction des positions et à leur négociation. Quels que soient les résultats des négociations engagées après Genève, qui risquent de durer, il reste que les différents acteurs africains partie prenante dans l'aventure de Cancún en ont tiré des leçons. Désormais, on ne peut plus parler des «producteurs de coton» ou des autres protagonistes africains (sociétés cotonnières, $\mathrm{ONG}$, États) de la même façon qu'avant cette prise de parole continentale

Denis Pesche Cirad, Montpellier Kako Nubukpo Cirad, Bamako

* Denis Pesche et Kako Nubukpo font partie de l'unité de recherche "Action collective, marchés et politiques publiques » du Centre de coopération internationale en recherche agronomique pour le développement (Cirad), respectivement à Montpellier et Bamako. Ils remercient Maurice Oudet (ABC Burkina) et Éric Hazard (Enda Diapol) pour leurs précieux commentaires et informations. 
1. La production mondiale de coton tourne autour de 20 millions de tonnes de coton fibre. Les grands pays producteurs sont la Chine, les États-Unis, l'Inde, le Pakistan et, dans une moindre mesure, l'Ouzbékistan et la Turquie. L'Union européenne et les pays du Sud-Est asiatique non producteurs sont les importateurs traditionnels, rejoints depuis peu par les grands pays producteurs d'Asie dont la consommation augmente plus vite que la production (Chine, Inde et Pakistan). Les principaux pays exportateurs de coton fibre sont les États-Unis, largement en tête avec près de $40 \%$ du marché, suivis par l'Afrique francophone (environ $15 \%$ ), l'Ouzbékistan (13\%) et l'Australie (10\%). 2. Voir L. Goreux, Le Coton après Cancún, OMC, mars 2004, p. 21, à consulter sur le site <http: / / www.oecd.org/ document/2/0,2340,en_2649_33711_32252418_1_1_1_1,00.html>; Secrétariat du Club du Sahel et de l'Afrique de l'Ouest, OCDE, «Événement spécial: le coton en Afrique de l'Ouest et du Centre et Cancún», note d'information sur les échanges entre acteurs à Paris, 2 septembre 2003.

3. Selon Oxfam International, les subventions américaines ont dépassé 3,9 milliards de dollars en 2001-2002, soit deux fois plus qu'en 1992. Cette somme dépasse le PIB du Burkina Faso (les trois quarts de ces subventions bénéficient aux $10 \%$ plus grands planteurs américains). Voir Oxfam International, Cultivating Poverty: The impact of US Cotton Subsidies on Africa, Oxfam Briefing Paper n 30, 2002. 4. L. Goreux, Le Coton après Cancún, op. cit., p. 11-12.

5. Depuis 2002, on constate une remontée des prix mais qui semble achevée avec des prévisions de baisse pour la prochaine saison 2004-2005. Voir les communiqués de presse de l'ICAC: <www.icac.org $>$.

6. À savoir concurrence, investissement, facilitation des échanges et transparence des marchés publics. Ces questions ont été un point de blocage central entre l'UE et les pays en développement. Voir A. Chetaille et K. Tavenier, Échec de la cinquième conférence ministérielle de l'OMC à Cancún: vers une crise du système commercial multilatéral?, Paris, Solagral, 2003.

7. C'est le cas, par exemple, du Canada, de l'Australie et de l'Argentine, connus comme des membres actifs du «groupe de Cairns"

8. «Du fait de l'apparition d'autres pouvoirs économiques (institutions internationales, firmes multinationales, marchés financiers), l'État apparaît plus décentré et fragmenté, réagissant ex post plutôt qu'ex ante, partageant son pouvoir de régulation avec d'autres acteurs ou d'autres entités", B. Hibou, «La "décharge", nouvel interventionnisme», Politique africaine, $\mathrm{n}^{\circ}$ 73, mars 1999, p. 6-15.

9. Comme le précise Jean Coussy, «Le scénario politique [du Nepad] est, lui, défini non par une analyse des dynamiques politiques africaines mais par l'affirmation que ces dynamiques vont s'inverser pour se conformer aux vœux des institutions internationales: rigueur financière, bonne gouvernance, démocratie, transparence, lutte contre la pauvreté et surveillance interafricaine mutuelle de la bonne exécution de ces réformes. L'avenir n'est donc pas défini par l'étude du présent mais par son refus. Ce qui est une démarche proprement utopique qui peut motiver les acteurs mais crée des risques élevés de non-réalisation », J. Coussy, «Le succès du Nepad, un paradoxe», Perspectives internationales, vol. 5 (1), p. 4-5.

10. Voir J. Stiglitz, La Grande Désillusion, Paris, Fayard, 2002. 11. Le G-21 regroupe l'Afrique du Sud, l'Argentine, la Bolivie, le Brésil, le Chili, la Chine, la Colombie, le Costa Rica, Cuba, l'Égypte, le Salvador, l'Équateur, le Guatemala, l'Inde, le Mexique, le Pakistan, le Paraguay, le Pérou, les Philippines, la Thailande et le Venezuela.

12. C'est notamment la thèse défendue par Éric Hazard dans De Bobo à Cancún, deux ans de campagne sur le coton: bilan et perspectives avant Genève, Dakar, Enda Diapol, 2004. 13. En octobre 2001, les cours du coton sont tombés à un niveau jamais atteint depuis les années 1930.

14. Voir la déclaration sur le site <http: / www.abcburkina.net/coton.htm>. Ce site, animé par Maurice Oudet au Burkina Faso, aura joué un rôle important pour faire connaître et soutenir l'initiative des producteurs africains de coton.

15. Enda Prospectives dialogues politiques (Sénégal) et Oxfam UK. Ce travail a débouché sur une étude tirée à 2500 exemplaires: P. Ton, Production cotonnière et conditions de vie en milieu rural en Afrique de l'Ouest, Dakar, Enda, coll. «Études et recherches », n² 219, 2001.

16. Le Réseau des organisations paysannes et de producteurs agricoles d'Afrique de l'Ouest a été créé en juillet 2000 et regroupe des plates-formes d'organisations paysannes de dix pays d'Afrique de l'Ouest. Son mandat général est de représenter ses membres au niveau régional et international. Voir le site <www.roppa-ao.org $>$.

17. Ce type de dynamique n'a rien de spécifiquement africain et a structuré la construction des organisations agricoles en France mais aussi aux États-Unis et dans beaucoup de pays industrialisés. Voir D. Pesche, Le Syndicalisme agricole spécialisé en France: entre la spécificité des intérêts et le besoin d'alliances, Paris, L'Harmattan, 2000.

18. African Growth and Opportunity Act: accords de coopération bilatéraux passés entre les États-Unis et des pays africains.

19. Le Roppa et l'ACA organiseront une conférence de presse commune en octobre 2002 pour soutenir la plainte déposée par le Brésil à l'ORD.

20. Oxfam International, Cultivating Poverty..., op. cit.

21. Son directeur, Nicolas Imboden, ancien négociateur suisse à l'OMC, jouera un rôle important dans la préparation du dossier coton et son introduction à l'OMC.

22. Principalement Oxfam et Enda Diapol qui noueront des alliances avec des organisations genevoises spécialisées 
dans l'appui aux pays en développement dans les négociations internationales (ICTSD, ACICI...). D'autres ONG et organisations de la société civile ont été impliquées, mais moins directement, avant Cancún.

23. Les éléments concernant la conférence de Cancún sont issus de E. Hazard, De Bobo à Cancún, deux ans de campagne sur le coton..., op. cit., d'un entretien avec l'auteur et de plusieurs entretiens réalisés par l'Observatoire du coton auprès de responsables agricoles africains présents à la conférence (pour une présentation de l'Observatoire du coton, voir <http: / / www.inter-reseaux.org/publications / graindesel/gds19/GDS19c6.htm>).

24. Les Africains disaient: «La culture du coton est une des rares activités pour lesquelles nous disposons d'un avantage comparatif et nous voulons exploiter cet avantage pour développer nos économies.» La réponse était « $\mathrm{Si}$ vous avez des problèmes avec le coton, faites autre chose», ce qui n'était pas encourageant. Voir L. Goreux, Le Coton après Cancún, op. cit.

25. A. Chetaille et K. Tavenier, Échec de la cinquième conférence ministérielle de l'OMC à Cancún..., op. cit.

26. Alors que, depuis Cancún, l'attitude américaine s'est opposée, dans un premier temps, à toute idée de concession commerciale sur le dossier coton, l'UE semble plus bienveillante et cherche à soutenir les filières cotonnières africaines.

27. Avec notamment l'émergence de la question du coton génétiquement modifié qui est actuellement largement cultivé aux EU, en Chine et de manière encore sporadique en Afrique (Afrique du Sud). Voir, à ce sujet, J. Pages, «Cotonniers génétiquement modifiés en Afrique: un besoin d'acquisition de connaissances », communication présentée au «Forum UE-Afrique sur le coton», Paris, 5-6 juillet 2004, à consulter sur le site <http: / / www.forum-coton.org/ indexflash.html >. Le Burkina Faso passe actuellement pour le pays qui facilite le plus la mise en place de biotechnologies dans la région. Voir K. Nubukpo, L'Avenir des filières cotonnières ouest-africaines: quelles perspectives après Cancún?, Paris, Cirad, Commission économique de la francophonie, 2004.

28. Roppa, Plan d'actions du Roppa et des OPPA pour le développement durable des filières africaines de coton, Cotonou, Roppa, 2004. «En menant des activités de plaidoyer et de lobbying sur la filière coton, le Roppa défend en fait l'ensemble des produits agricoles concernés par les échanges internationaux: céréales, oléagineux, café, cacao, bétail, viande, lait... Ainsi, le premier enjeu pour le Roppa est d'établir une stratégie de défense de toutes les spéculations et produits agricoles majeurs des exploitations familiales", Roppa, Initiative paysanne en faveur du développement de la filière coton en Afrique: contribution provisoire pour discussion et débat, Ouagadougou, Roppa, 2004.

29. «La période actuelle est pleine d'incertitudes. Sans régulation équitable des marchés internationaux et sans organisation solide des acteurs, les libéralisations en cours présentent des risques élevés. La modernisation et la structuration des agricultures familiales constituent donc des facteurs incontournables pour relancer des dynamiques économiques durables leur permettant d'éviter de nouveaux reculs dans leur développement. » Voir J.-C. Devèse, «Les agricultures familiales cotonnières entre modernisation et marginalisation », communication présentée au Forum UE-Afrique sur le coton. Sur l'enjeu de politiques agricoles renouvelées, voir P.-M. Bosc, J. Berthomé, B. Losch et M.-R. Mercoiret, «Le grand saut des organisations de producteurs agricoles africaines: de la protection sous tutelle à la mondialisation", Revue internationale d'Économie sociale, $\mathrm{n}^{\circ}$ 285, 2002, p. 47-62. 30. É. Hazard, De Bobo à Cancún, deux ans de campagne sur le coton..., op. cit.

31. Le Bénin, pays coordonnateur de la démarche coton, ne donne pas forcément l'exemple d'un État assurant de manière impartiale les arbitrages et le respect des engagements pris dans le cadre interprofessionnel de gestion de la filière coton. Comme le suggère une analyse du Roppa sur les interprofessions existantes dans les filières cotonnières: «Les rôles d'administration et de gestion internes de la filière, notamment par la régulation des relations entre acteurs, d'arbitrage des conflits, d'appuis au développement de la filière ne sont pas encore effectifs du fait d'une absence de définition et de répartition claires des rôles et responsabilités des acteurs, de capacités encore faibles des acteurs pour jouer leur rôle respectif. Il s'y ajoute que les rôles de tutelle des États sont encore peu effectifs.» Voir Roppa, Plan d'actions du Roppa et des OPPA..., op. cit. 32. M. Offerlé, Sociologie des groupes d'intérêts, Paris, Montchrestien, 1994. 